\title{
Oxidative stabilization of corn oil with spinach extract
}

\author{
M. Tehseen ${ }^{1}$, S. Hina ${ }^{2 *}$, Alim-un-Nisa ${ }^{2}$ and A. Ahmad ${ }^{3}$ \\ ${ }^{1}$ Institute of Chemistry, University of the Punjab, Quaid-i-Azam Campus, Lahore, Pakistan \\ ${ }^{2}$ Food \& Biotechnology Research Centre, PCSIR Laboratories Complex, Ferozepur Road, Lahore, Pakistan \\ ${ }^{3}$ Land Resources Research Institute, National Agricultural Research Centre, Islamabad- 45500, Pakistan
}

Received: 29 November 2018

Revised: 10 January 2019

Accepted: 06 May 2019

DOI: https://doi.org/10.3329/bjsir.v54i4.44572

\begin{abstract}
The present study was aimed to analyze the antioxidant activity of spinach (Spinacia oleracea) extract in oxidative stabilization of refined, bleached and de-odorized (RBD) corn oil (CO). Corn oil was supplemented with three different concentrations 600,1200 and $1800 \mathrm{ppm}$ of methanolic spinach extract and then stored at $25^{\circ} \mathrm{C}$ and $60^{\circ} \mathrm{C}$. Different oxidation measuring parameters such as peroxide value (PV), free fatty acid (FFA) value, iodine value (IV), conjugated dienes (CD), conjugated trienes (CT) and para-anisidine value (PAV) were examined. The potency of spinach extract as natural antioxidant was found to be as effective as the synthetic antioxidants BHA and BHT used as standards and highest stabilization was observed in the order CO-1800 $>$ CO-1200 $>$ CO-BHT $>$ $\mathrm{CO}-\mathrm{BHA}>\mathrm{CO}-600>\mathrm{CO}-$ control. The results showed a significant effect of spinach extract in enhancing the oxidative stability of corn oil.
\end{abstract}

Keywords: Spinach extract; Corn oil; Oxidation; Stabilization; BHA and BHT

\section{Introduction}

The shelf life of plant derived oils is greatly dependent on their oxidative stability which is a serious concern for their practical applications (Gertz et al., 2000). Oxidative deterioration of these oils results in the formation of free radicals and reactive oxygen species badly effecting not only the quality of these oils but also posing serious health threats (Nosratpour et al., 2017; Kanner, 2007). In order to enhance the oxidative stability of oils various methods are employed such as genetic modifications, compositional changes and use of additive materials such as antioxidants (Shahidi and Zhong, 2010). Use of many synthetic antioxidants such as butylatedhydroxyanisole (BHA), butylatedhydroxytoluene (BHT), ter-butyl hydro quinone (TBHQ) is common as potential inhibitors of lipid peroxidation in the oils and fats (Aluyor and Ori-Jesu, 2008; Ullah et al., 2003; Azeez et al., 2013). However, the probable health deleterious effects of these synthetic antioxidants limit their use in the edible oils.
Exploration of antioxidants of natural origin is increasing now a days (Rajendran et al., 2014; Augustyniak et al., 2010). Owing to the health promoting properties, natural antioxidants obtained mainly from fruits and vegetables are gaining the attention (Aladedunye, 2014; Li et al., 2014). Many studies revealed that people consuming more fruits and vegetables enriched with the natural antioxidants such as flavonoids, polyphenols, vitamin $\mathrm{C}$ are at a lower risk of chronic and cardiovascular diseases (Kris-Etherton et al., 2002). Various plant based natural antioxidants are explored and well characterized and their role as a potential substitute of synthetic antioxidants has also been well proven (Tundis et al., 2017). The use of natural antioxidants from different plant sources in stabilization of many vegetable oils has also been well studied (Redondo-Cuevas et al., 2017; Aydeniz and Yilmaz, 2016; Taghvaei and Jafari, 2015).

Corn oil is commonly used both for edible purposes as well 
as in many industrial applications. Presence of large content of unsaturated fatty acids and residual tocopherol contents after refining makes it more vulnerable to oxidation (Naz et al., 2005; Naz et al., 2004). Several investigations using this potential oxidative substrate was carried out to explore the antioxidant effectiveness of many plant extracts. In a study, Navas et al. (2006) reported the effectiveness of black tea, garlic and onion on stability of corn oil at different temperatures. In addition to plant extracts, seed oil of black cumin and coriander when added to corn oil was also proved to be effective at $60^{\circ} \mathrm{C}$ temperature for 15 days storage (Ramadan and Wahdan 2012). Thermal stability of corn oil was assessed at different heating temperatures using thyme flower extracts and it was shown that thyme flower enhanced the stability of corn oil (Karoui et al., 2011). Sultana et al. (2008) investigated the antioxidative efficacy of different agro-waste extracts and found them as potent source for enhancing the oxidative stability of corn oil. In another study, they utilize the corncob extract for the stabilization of corn oil under microwave heating and found good results (Sultana et al., 2007). Anwar et al. (2003) observed the antioxidant potential of methanolic extracts of rice bran, guava leaf, roasted wheat germ, rosemary, coffee beans, basil and peppermint in stabilizing refined corn oil at heating conditions of $65^{\circ} \mathrm{C}$ concluding all these extracts to be very effective as natural antioxidants. Some plant extracts were also investigated for enhancing the oxidative stability of corn oil under high pressure accelerated oxidation conditions (Bandak et al., 2011).

Spinach(Spinacia oleracea $)$ is one of the most peculiar green leafy vegetable famous for its nutritional contents and also enriched with a number of phytochemical compounds giving it superb antioxidant activities (Ko et al., 2014; Lee et al., 2002). Utilization of spinach extract in oxidative stabilization of refined, bleached, and de-odorized corn oil was investigated in the present work. The results from all the parameters used to estimate the oxidation of oil clearly depicts the potency of spinach extract as a natural antioxidant in improving the stability of corn oil.

\section{Materials and methods}

\section{Sample}

Samples of refined, bleached and de-odorized (RBD) corn oil were procured locally.

\section{Preparation of spinach extract}

Spinach samples were first washed thoroughly to remove soil and then macerated. The crushed sample $(10 \mathrm{~g})$ was then extracted using $25 \mathrm{ml}$ methanol by shaking well at room temperature overnight. The pure extract was collected after subjecting to Soxhlet apparatus for about $3 \mathrm{hrs}$. The extract was dried and measuring yield stored at $4^{\circ} \mathrm{C}$ until used for further analysis.

\section{Estimation of antioxidant potential of spinach extract}

Antioxidant potential of spinach extract was estimated by total phenolic contents, DPPH free radical scavenging activity, $\beta$-carotene linoleate method, ferric reducing antioxidant power and ferric thiocyanate method as described in our previously reported work (Tehseen et al., 2014). Methanolic extracts of canal water irrigated wavy leaves spinach sample has shown the maximum antioxidant potential which are employed for this study.

\section{Estimation of stability of corn oil using spinach extract}

The prepared spinach extract was added to RBD corn oil at three different concentrations 600ppm, 1200ppm and $1800 \mathrm{ppm}$. Samples were agitated vigorously for uniform mixing. Standard samples were prepared using synthetic antioxidants BHT and BHA at their safe limits of 200ppm. Beside control samples without any antioxidant was also taken. All these stabilized and control samples in triplicate were stored in amber glass bottles at $25^{\circ} \mathrm{C}$ and $60^{\circ} \mathrm{C}$ for 40 days and then analyzed further for oxidative degradation. Peroxide value (PV), Free fatty acid Value (FFA), Iodine value (IV), Conjugated dienes (CD) and trienes (CT) of both stabilized and control samples were measured according to the standard methods of IUPAC (IUPAC, 1987)and p-Anisidine value was analyzed as described by Saha et al. (2008).

\section{Statistical analysis}

All the oxidation parameters were performed in triplicate and data was analyzed statistically by using one-way ANOVA (SPSS ver. 8.0) and significant differences $(\mathrm{P}<0.05)$ among different parameters were calculated (Steel et al., 1997).

\section{Results and discussion}

\section{Peroxide value (PV)}

Peroxide value represent the extent of formation of primary oxidation products during oxidative degradation of oils and fats. Hence the measurement of peroxide value can be used to depict how much an antioxidant inhibit the formation of these oxidation products. Peroxide values of corn oil stabilized with spinach extract, BHA, BHT and control at $25^{\circ} \mathrm{C}$ and $60^{\circ} \mathrm{C}$ for a storage period of 40 days is presented in Table I and II. The amber glass bottle was used for storage 
Table I. Relative effect of storage conditions on PV of corn oil at $25^{\circ} \mathrm{C}$

\begin{tabular}{ccccccc}
\hline $\begin{array}{c}\text { Storage } \\
\text { time } \\
\text { (Days) }\end{array}$ & CO-Ctrl & CO-600 & CO-1200 & CO-1800 & CO-BHA & CO-BHT \\
\cline { 2 - 7 } & $0.21 \pm 0.06$ & $0.21 \pm 0.06$ & $0.21 \pm 0.06$ & $0.21 \pm 0.06$ & $0.21 \pm 0.06$ & $0.21 \pm 0.06$ \\
10 & $5.12 \pm 0.12$ & $3.41 \pm 0.18$ & $1.69 \pm 0.12$ & $1.12 \pm 0.09$ & $1.61 \pm 0.09$ & $1.09 \pm 0.13$ \\
20 & $21.72 \pm 0.17$ & $10.09 \pm 0.12$ & $7.23 \pm 0.16$ & $5.81 \pm 0.13$ & $6.02 \pm 0.11$ & $5.78 \pm 0.08$ \\
30 & $32.61 \pm 0.09$ & $14.62 \pm 0.09$ & $10.34 \pm 0.13$ & $9.33 \pm 0.08$ & $10.35 \pm 0.08$ & $9.32 \pm 0.11$ \\
40 & $46.02 \pm 0.07$ & $23.55 \pm 0.16$ & $19.26 \pm 0.11$ & $16.2 \pm 0.06$ & $18.94 \pm 0.13$ & $15.61 \pm 0.11$ \\
\hline
\end{tabular}

Table II. Relative effect of storage conditions on PV of Corn oil at $60^{\circ} \mathrm{C}$

\begin{tabular}{ccccccc}
\hline $\begin{array}{c}\text { Storage } \\
\text { time } \\
\text { (Days) }\end{array}$ & CO-Ctrl & CO-600 & CO-1200 & CO-1800 & CO-BHA & CO-BHT \\
\cline { 2 - 7 } & $0.21 \pm 0.06$ & $0.21 \pm 0.06$ & $0.21 \pm 0.06$ & $0.21 \pm 0.06$ & $0.21 \pm 0.06$ & $0.21 \pm 0.06$ \\
10 & $10.61 \pm 0.03$ & $3.96 \pm 0.11$ & $2.68 \pm 0.09$ & $1.86 \pm 0.05$ & $2.69 \pm 0.21$ & $1.82 \pm 0.04$ \\
20 & $36.73 \pm 0.05$ & $12.01 \pm 0.05$ & $7.91 \pm 0.16$ & $6.42 \pm 0.03$ & $7.73 \pm 0.19$ & $6.39 \pm 0.09$ \\
30 & $56.54 \pm 0.03$ & $17.22 \pm 0.09$ & $14.53 \pm 0.13$ & $10.67 \pm 0.06$ & $12.56 \pm 0.09$ & $10.62 \pm 0.16$ \\
40 & $69.76 \pm 0.08$ & $28.07 \pm 0.07$ & $23.46 \pm 0.08$ & $18.06 \pm 0.07$ & $19.88 \pm 0.16$ & $17.92 \pm 0.08$ \\
\hline
\end{tabular}

Free fatty acid value (FFA)

Table III. Relative effect of storage conditions on FFA of Corn oil at $25^{\circ} \mathrm{C}$

\begin{tabular}{|c|c|c|c|c|c|c|}
\hline \multirow{2}{*}{$\begin{array}{l}\text { Storage } \\
\text { time } \\
\text { (Days) }\end{array}$} & \multicolumn{6}{|c|}{ Free fatty acid value (FFA) $(\%$ oleic acid $)$} \\
\hline & $\mathrm{CO}-\mathrm{Ctrl}$ & CO-600 & CO-1200 & CO-1800 & CO-BHA & CO-BHT \\
\hline 0 & $0.02 \pm 0.01$ & $0.02 \pm 0.01$ & $0.02 \pm 0.01$ & $0.02 \pm 0.01$ & $0.02 \pm 0.01$ & $0.02 \pm 0.01$ \\
\hline 10 & $0.14 \pm 0.04$ & $0.05 \pm 0.08$ & $0.03 \pm 0.06$ & $0.02 \pm 0.04$ & $0.03 \pm 0.01$ & $0.02 \pm 0.07$ \\
\hline 20 & $0.19 \pm 0.03$ & $0.06 \pm 0.08$ & $0.04 \pm 0.04$ & $0.02 \pm 0.02$ & $0.04 \pm 0.03$ & $0.03 \pm 0.06$ \\
\hline 30 & $0.24 \pm 0.01$ & $0.09 \pm 0.06$ & $0.07 \pm 0.03$ & $0.04 \pm 0.03$ & $0.06 \pm 0.02$ & $0.05 \pm 0.05$ \\
\hline 40 & $0.35 \pm 0.02$ & $0.14 \pm 0.09$ & $0.10 \pm 0.04$ & $0.06 \pm 0.02$ & $0.09 \pm 0.01$ & $0.07 \pm 0.02$ \\
\hline
\end{tabular}

to prevent UV light deterioration as UV light may enhance oxidation of lipid that destabilized the activity. The result shows a significant reduction in peroxide values of the samples with spinach extract and standard antioxidants as compared to control. Spinach extract at maximum concentration of $1800 \mathrm{ppm}$ showed highest reduction in peroxide value $(\mathrm{P}<0.05)$ comparable with that of the standard antioxidants which indicates the efficacy of spinach extract in stabilization of corn oil at both storage temperatures. Sultana et al. (2007) reported the efficacy of corncob extracts in lowering the PVs of corn oil during microwave incubation. Navas et al. (2006) investigated the efficacy of black tea, onion and garlic extracts in stabilizing corn oil at heating temperatures of 55 and $140^{\circ} \mathrm{C}$. Black tea was found to be most effective in enhancing corn oil stability. They also showed that determining PVs is rather less feasible at high heating temperatures as the peroxides formed earlier decompose or evaporate at high temperatures.

Free fatty acid value (FFA)

Free fatty acids are the degradation products formed by hydrolysis of fats or oils. They are more prone to oxidation and causes rancidity of fats and oils hence representing a key feature in predicting the extent of deterioration. Increase in FFA value during storage indicates more oxidative damage. In the present study FFA values of RBD corn oil stabilized with synthetic antioxidants and spinach extract was estimated at $25^{\circ} \mathrm{C}$ and $60^{\circ} \mathrm{C}$ 
Table IV. Relative effect of storage conditions on FFA of corn oil at $60^{\circ} \mathrm{C}$

\begin{tabular}{ccccccc}
\hline $\begin{array}{c}\text { Storage } \\
\text { time } \\
\text { (Days) }\end{array}$ & CO-Ctrl & CO-600 & CO-1200 & CO-1800 & CO-BHA & CO-BHT \\
\cline { 2 - 6 } & $0.02 \pm 0.01$ & $0.02 \pm 0.01$ & $0.02 \pm 0.01$ & $0.02 \pm 0.01$ & $0.02 \pm 0.01$ & $0.02 \pm 0.01$ \\
0 & $0.06 \pm 0.07$ & $0.05 \pm 0.05$ & $0.03 \pm 0.05$ & $0.04 \pm 0.05$ & $0.03 \pm 0.03$ \\
10 & $0.17 \pm 0.04$ & $0.09 \pm 0.09$ & $0.07 \pm 0.03$ & $0.04 \pm 0.03$ & $0.05 \pm 0.03$ & $0.06 \pm 0.05$ \\
20 & $0.21 \pm 0.06$ & $0.13 \pm 0.07$ & $0.08 \pm 0.02$ & $0.07 \pm 0.05$ & $0.07 \pm 0.01$ & $0.08 \pm 0.04$ \\
30 & $0.33 \pm 0.02$ & $0.18 \pm 0.06$ & $0.12 \pm 0.04$ & $0.10 \pm 0.04$ & $0.09 \pm 0.02$ & $0.12 \pm 0.03$ \\
40 & $0.42 \pm 0.03$ & & & &
\end{tabular}

Table V. Relative effect of storage conditions on IV of corn oil at $25^{\circ} \mathrm{C}$

\begin{tabular}{ccccccc}
\hline $\begin{array}{c}\text { Storage } \\
\text { time } \\
\text { (Days) }\end{array}$ & CO-Ctrl & CO-600 & CO-1200 & CO-1800 & CO-BHA & CO-BHT \\
\cline { 2 - 6 } & $142 \pm 2.01$ & $142 \pm 2.01$ & $142 \pm 2.01$ & $142 \pm 2.01$ & $142 \pm 2.01$ & $142 \pm 2.01$ \\
0 & $140 \pm 2.02$ & $140 \pm 2.22$ & $141 \pm 2.07$ & $141 \pm 2.06$ & $141 \pm 2.09$ & $142 \pm 1.09$ \\
10 & $138 \pm 1.09$ & $139 \pm 2.06$ & $139 \pm 2.06$ & $141 \pm 2.02$ & $140 \pm 1.14$ & $141 \pm 1.14$ \\
30 & $133 \pm 1.06$ & $138 \pm 2.06$ & $138 \pm 2.87$ & $140 \pm 2.08$ & $140 \pm 1.19$ & $141 \pm 2.01$ \\
40 & $121 \pm 2.02$ & $137 \pm 2.04$ & $138 \pm 1.98$ & $139 \pm 1.94$ & $139 \pm 2.12$ & $140 \pm 2.09$ \\
\hline
\end{tabular}

for 40 days storage (Table III and IV). The increase in FFA values was less in the samples stabilized with spinach extract which is comparable with the synthetic antioxidants. Spinach extract was found efficient to slow down the rate of formation of FFA as compared to the control samples which showed maximum increase in FFA. A significant variation $(\mathrm{p} \leq 0.05)$ in FFA contents of both stabilized and control samples was observed. The results are in close agreement with the previous studies on stabilizing the sunflower oil with extracts of waste cake which showed significant reduction in FFA values (Abd-El-Ghany et al., 2010). Ali et al. (2016) also studied the FFA values as an indicative tool for depicting the oxidative stabilization of cooking oil blends with added Eucalyptus citriodora leaf extract and showed that there is less increase in value of FFA during the induction period.

\section{Iodine value (IV)}

Degree of unsaturation of an oil or fat is measured in terms of its iodine value. Oil and fats with more unsaturation are more vulnerable to oxidation and become rancid. A significant decrease in IV of both stabilized and non-stabilized (control) corn oil samples was observed during the storage period and the maximum decrease was recorded after a 40 day induction period. However, this decrease in oil unsaturation was more in control corn oil samples. The results presented in Table V and VI indicate that a relatively small decrease in oil unsaturation was recorded in corn oil that were stabilized with spinach extract which is similar to that stabilized with BHT and BHA. These IVs for corn oil in the present study were correlated to the values reported in sunflower oil blended with olive waste cake extract (Abd-El-Ghany et al., 2010) and also in agreement with the observations that IV of blend oil samples show less decrease when stabilized with Eucalyptus citriodora leaf extract (Ali et al., 2016).

\section{Conjugated dienes and trienes value (CD and CT)}

Oxidation process of oils and fats results in the formation of conjugated dienes and trienes due to shifting of double bonds and showing a particular absorbance at 232 and $268 \mathrm{~nm}$ respectively. Hence, both these values directly correlate with the extent of oxidation. Oxidative stability of oils decreases, if the $\mathrm{CD}$ and $\mathrm{CT}$ values increases in consequence of oxidative degradation. $\mathrm{CD}$ and $\mathrm{CT}$ values of corn oil sample stabilized with spinach extract, BHA, BHT and control is shown in Table VII, VIII, IX and $\mathrm{X}$ respectively. Supplementation with the spinach extract shows a significant reduction in $\mathrm{CD}$ and $\mathrm{CT}$ values as compared to control and the effect is also comparable to standard antioxidants used. The present findings are in close agreement with the other studies performed to evaluate the CD and CT contents of oils stabilized with different natural plant extracts. Sultana et al. (2008) measured CD and CT contents of corn oil stabilized with agro-wastes of different plant sources and showed that 
Table VI. Relative effect of storage conditions on IV of corn oil at $60^{\circ} \mathrm{C}$

\begin{tabular}{|c|c|c|c|c|c|c|}
\hline \multirow{2}{*}{$\begin{array}{l}\text { Storage } \\
\text { Time } \\
\text { (Days) }\end{array}$} & \multicolumn{6}{|c|}{ Iodine value (IV) } \\
\hline & $\mathrm{CO}-\mathrm{Ctrl}$ & CO-600 & CO-1200 & CO-1800 & CO-BHA & CO-BHT \\
\hline 0 & $142 \pm 2.01$ & $142 \pm 2.01$ & $142 \pm 2.01$ & $142 \pm 2.01$ & $142 \pm 2.01$ & $142 \pm 2.01$ \\
\hline 10 & $139 \pm 1.09$ & $140 \pm 2.09$ & $141 \pm 2.06$ & $141 \pm 2.08$ & $141 \pm 2.12$ & $140 \pm 1.08$ \\
\hline 20 & $135 \pm 1.07$ & $136 \pm 2.06$ & $139 \pm 2.09$ & $140 \pm 1.77$ & $139 \pm 1.13$ & $139 \pm 1.29$ \\
\hline 30 & $129 \pm 1.06$ & $132 \pm 2.07$ & $138 \pm 2.01$ & $138 \pm 1.96$ & $136 \pm 118$ & $138 \pm 1.18$ \\
\hline 40 & $118 \pm 2.00$ & $126 \pm 1.19$ & $130 \pm 2.03$ & $136 \pm 2.09$ & $132 \pm 2.03$ & $135 \pm 2.04$ \\
\hline
\end{tabular}

Table VII. Relative effect of storage conditions on CD value of corn oil at $25^{\circ} \mathrm{C}$

\begin{tabular}{ccccccc}
\hline $\begin{array}{c}\text { Storage } \\
\text { time } \\
\text { (Days) }\end{array}$ & CO-Ctrl & CO-600 & CO-1200 & CO-1800 & CO-BHA & CO-BHT \\
\cline { 2 - 6 }$y$ & $1.62 \pm 0.03$ & $1.62 \pm 0.10$ & $1.62 \pm 0.03$ & $1.62 \pm 0.03$ & $1.62 \pm 0.03$ & $1.62 \pm 0.03$ \\
10 & $3.96 \pm 0.10$ & $2.91 \pm 0.82$ & $2.44 \pm 0.05$ & $2.13 \pm 0.11$ & $2.48 \pm 0.14$ & $2.03 \pm 0.21$ \\
20 & $8.32 \pm 0.08$ & $4.83 \pm 0.96$ & $4.51 \pm 0.07$ & $4.18 \pm 0.13$ & $4.52 \pm 0.11$ & $4.16 \pm 0.17$ \\
30 & $11.62 \pm 0.06$ & $7.72 \pm 0.77$ & $7.42 \pm 0.06$ & $6.91 \pm 0.09$ & $7.13 \pm 0.09$ & $6.73 \pm 0.19$ \\
40 & $15.71 \pm 0.07$ & $8.36 \pm 0.80$ & $8.01 \pm 0.02$ & $7.78 \pm 0.12$ & $7.98 \pm 0.12$ & $7.52 \pm 0.16$ \\
\hline
\end{tabular}

Table VIII. Relative effect of storage conditions on CD value of corn oil at $60^{\circ} \mathrm{C}$

\begin{tabular}{ccccccc}
\hline $\begin{array}{c}\text { Storage } \\
\text { time } \\
\text { (Days) }\end{array}$ & CO-Ctrl & CO-600 & CO-1200 & CO-1800 & CO-BHA & CO-BHT \\
\cline { 2 - 6 } & $1.62 \pm 0.03$ & $1.62 \pm 0.03$ & $1.62 \pm 0.03$ & $1.62 \pm 0.03$ & $1.62 \pm 0.03$ & $1.62 \pm 0.03$ \\
10 & $4.24 \pm 0.04$ & $3.49 \pm 0.05$ & $3.11 \pm 0.04$ & $2.83 \pm 0.13$ & $3.01 \pm 0.15$ & $2.81 \pm 0.11$ \\
20 & $9.82 \pm 0.15$ & $6.05 \pm 0.11$ & $5.82 \pm 0.06$ & $5.39 \pm 0.11$ & $5.81 \pm 0.13$ & $5.32 \pm 0.13$ \\
30 & $13.53 \pm 0.10$ & $9.86 \pm 0.09$ & $9.27 \pm 0.05$ & $8.98 \pm 0.13$ & $9.14 \pm 0.08$ & $8.84 \pm 0.09$ \\
40 & $17.65 \pm 0.09$ & $11.23 \pm 0.08$ & $10.93 \pm 0.04$ & $10.31 \pm 0.12$ & $10.65 \pm 0.11$ & $10.09 \pm 0.06$ \\
\hline
\end{tabular}

Table IX. Relative effect of storage conditions on CT values of corn oil at $25^{\circ} \mathrm{C}$

\begin{tabular}{ccccccc}
\hline $\begin{array}{c}\text { Storage } \\
\text { time } \\
\text { (Days) }\end{array}$ & CO-Ctrl & CO-600 & CO-1200 & CO-1800 & CO-BHA & CO-BHT \\
\cline { 2 - 7 } & $0.38 \pm 0.11$ & $0.38 \pm 0.11$ & $0.38 \pm 0.11$ & $0.38 \pm 0.11$ & $0.38 \pm 0.11$ & $0.38 \pm 0.11$ \\
0 & $1.92 \pm 0.09$ & $1.62 \pm 0.09$ & $1.08 \pm 0.13$ & $0.73 \pm 0.07$ & $1.01 \pm 0.06$ & $0.72 \pm 0.06$ \\
10 & $2.61 \pm 0.12$ & $2.16 \pm 0.06$ & $2.01 \pm 0.14$ & $0.93 \pm 0.11$ & $1.82 \pm 0.02$ & $0.91 \pm 0.07$ \\
30 & $3.84 \pm 0.14$ & $3.27 \pm 0.07$ & $3.11 \pm 0.12$ & $1.27 \pm 0.13$ & $2.36 \pm 0.13$ & $1.23 \pm 0.12$ \\
40 & $5.09 \pm 0.15$ & $4.35 \pm 0.13$ & $3.86 \pm 0.11$ & $1.49 \pm 0.09$ & $2.87 \pm 0.16$ & $1.46 \pm 0.13$ \\
\hline
\end{tabular}


Table X. Relative effect of storage conditions on CT values of corn oil at $60^{\circ} \mathrm{C}$

\begin{tabular}{ccccccc}
\hline $\begin{array}{c}\text { Storage } \\
\text { time } \\
(\text { Days })\end{array}$ & CO-Ctrl & CO-600 & CO-1200 & CO-1800 & CO-BHA & CO-BHT \\
\cline { 2 - 6 } & $0.38 \pm 0.11$ & $0.38 \pm 0.11$ & $0.38 \pm 0.11$ & $0.38 \pm 0.11$ & $0.38 \pm 0.11$ & $0.38 \pm 0.11$ \\
0 & $1.76 \pm 0.12$ & $1.61 \pm 0.09$ & $0.81 \pm 0.17$ & $1.09 \pm 0.12$ & $0.76 \pm 0.14$ \\
10 & $2.07 \pm 0.16$ & $2.91 \pm 0.17$ & $2.73 \pm 0.07$ & $1.09 \pm 0.12$ & $2.16 \pm 0.19$ & $0.98 \pm 0.12$ \\
20 & $3.56 \pm 0.17$ & $4.87 \pm 0.09$ & $3.56 \pm 0.02$ & $1.46 \pm 0.15$ & $2.54 \pm 0.09$ & $1.32 \pm 0.17$ \\
30 & $4.92 \pm 0.12$ & $5.29 \pm 0.08$ & $4.01 \pm 0.12$ & $1.88 \pm 0.14$ & $3.01 \pm 0.16$ & $1.63 \pm 0.13$ \\
\hline 0 & $6.13 \pm 0.09$ & & & & & \\
\hline
\end{tabular}

Table XI. Relative effect of storage conditions on $p$-anisidine value of corn oil at $25^{\circ} \mathrm{C}$

\begin{tabular}{ccccccc}
\hline $\begin{array}{c}\text { Storage } \\
\text { Time } \\
\text { (Days) }\end{array}$ & CO-Ctrl & CO-600 & CO-1200 & CO- 1800 & CO-BHA & CO-BHT \\
\hline & $1.04 \pm 0.40$ & $1.04 \pm 0.40$ & $1.04 \pm 0.40$ & $1.04 \pm 0.40$ & $1.04 \pm 0.40$ & $1.04 \pm 0.40$ \\
10 & $3.93 \pm 0.37$ & $2.82 \pm 0.12$ & $2.31 \pm 0.52$ & $1.98 \pm 0.31$ & $2.02 \pm 0.92$ & $1.88 \pm 0.19$ \\
20 & $5.35 \pm 0.31$ & $3.94 \pm 0.09$ & $3.24 \pm 0.61$ & $2.88 \pm 0.52$ & $2.99 \pm 0.83$ & $2.74 \pm 0.17$ \\
30 & $9.21 \pm 0.35$ & $5.32 \pm 0.10$ & $4.21 \pm 0.59$ & $3.78 \pm 0.55$ & $3.89 \pm 0.86$ & $3.61 \pm 0.09$ \\
40 & $11.97 \pm 0.39$ & $7.93 \pm 0.08$ & $6.09 \pm 0.53$ & $5.62 \pm 0.39$ & $5.97 \pm 0.56$ & $5.08 \pm 0.15$ \\
\hline
\end{tabular}

Table XII. Relative effect of storage conditions on $p$-anisidine value of corn oil at $60^{\circ} \mathrm{C}$

\begin{tabular}{ccccccc}
\hline $\begin{array}{c}\text { Storage } \\
\text { time } \\
\text { (Days) }\end{array}$ & CO-Ctrl & CO-600 & CO-1200 & CO- 1800 & CO-BHA & CO-BHT \\
\cline { 2 - 6 } & $1.04 \pm 0.40$ & $1.04 \pm 0.40$ & $1.04 \pm 0.40$ & $1.04 \pm 0.40$ & $1.04 \pm 0.40$ & $1.04 \pm 0.40$ \\
0 & $3.87 \pm 0.09$ & $3.37 \pm 0.93$ & $2.91 \pm 0.23$ & $3.08 \pm 0.62$ & $2.30 \pm 0.37$ \\
10 & $4.31 \pm 0.32$ & $5.89 \pm 0.12$ & $5.21 \pm 0.48$ & $4.83 \pm 0.29$ & $4.92 \pm 0.65$ & $4.62 \pm 0.33$ \\
20 & $8.42 \pm 0.34$ & $7.31 \pm 0.23$ & $7.08 \pm 0.59$ & $6.78 \pm 0.32$ & $6.89 \pm 0.49$ & $6.17 \pm 0.39$ \\
30 & $13.16 \pm 0.35$ & $10.62 \pm 0.29$ & $10.06 \pm 0.62$ & $9.62 \pm 0.25$ & $9.78 \pm 0.53$ & $9.21 \pm 0.43$ \\
40 & $18.69 \pm 0.38$ & & & & & \\
\hline
\end{tabular}

about $71 \%$ and $59 \%$ reduction occur. In another study they tested the efficacy of corncob extract in stabilization of corn oil and observed about $45 \%$ and $39 \%$ inhibition in CD and CT values as compared to control (Sultana et al., 2007). Siddiq et al. (2005) evaluated the antioxidant potential of methanolicextractof Moringaoleifera leave in sunflower oil stabilization by measuring both $\mathrm{CD}$ and $\mathrm{CT}$ values with a significant decline in the rise of both these values. CD and CT Parameters as indicative of the oxidative degradation was also investigated for sunflower oil stability using garlic extract (Iqbal and Bhanger 2007).

\section{p-anisidine value}

Many non-volatile carbonyl compounds mostly aldehydes formed as secondary oxidation products of lipid peroxidation are estimated by $p$-anisidine values. The concentration of aldehydes directly correlates with oxidative degradation of the oils, greater amount of these aldehydes in the oils corresponds to less oxidative stability. These aldehydes react with the $p$-anisidine reagent to form a coloured product, the absorbance of which is measured at $350 \mathrm{~nm}$ on spectrophotometer. The $p$-anisidine values of the corn oil stabilized with spinach extract, BHT, BHA and 
control is shown in Table XI and XII. The results showed that the corn oil with the added spinach extract inhibited the rise in $p$-anisidine value as compared to control both at ambient and accelerated storage temperature. The rate of formation of carbonyl compounds is high in control sample as compared to those with the added spinach extract and standard antioxidants. The maximum reduction in $p$-anisidine value was observed for the spinach extract at CO-1800 concentration and these are also consistent with the decline in $p$-anisidine values for the samples with standard BHA and BHT. Sultana et al. (2007) represented the $p$-ansidine values for corn oil samples stabilized with corncob methanolic extract, BHT, and control. The corncob extract at $1000 \mathrm{ppm}$ concentration showed a significant decrease $41.8 \%$ as compared to control. The extracts of agro wastes of different plant materials was assessed in stabilizing corn oil and the $p$-anisidine values measured showed a reduction up to $59 \%$ with the added extract (Sultana et al., 2008). $p$-Anisidine values of soyabean oil also show a decline up to $24 \%$ when stabilized with the rice bran extract at $60^{\circ} \mathrm{C}$ (Chatha et al., 2006).

\section{Conclusion}

Vegetable oils are more prone to oxidative deterioration during storage, hence use of synthetic antioxidants is common to enhance their oxidative stability. However, there is space for work on exploration of natural antioxidants which can be used to enhance the oxidative stabilization of vegetable oils. The present research is aimed to investigate the efficacy of spinach extract in stabilization of corn oil. The results of different oxidation parameters depicted that spinach is a good source of antioxidants and more potent in enhancing the oxidative stability of corn oil. Further investigations in the subject matter is needed to develop useful antioxidants of natural origin and to enhance their practical applicability in vegetable oils and other food products.

\section{References}

Ali S, Chatha SAS, Ali Q, Hussain AI, Hussain SM and Perveen R (2016), Oxidative stability of cooking oil blend stabilized with leaf extract of Eucalyptus citriodora, Int J Food Prop. 19: 1556-1565. DOI: org/10.1080/ 10942912. 2015.1047514

Aydeniz B and Yilmaz E (2016), Performance of different natural antioxidant compounds in frying oil, Food Tech Biotech. 54(1): 21-30. DOI: org/10.17113/ftb.54.01.16.4109

Aladedunye FA (2014), Natural antioxidants as stabilizers of frying oils, Eur J Lipid Sci Tech. 116: 688-706.
Azeez OT, Ejeta KO, Frank EO and Gerald NE (2013), Effects of antioxidants on the oxidative stability of vegetable oil at elevated temperature, Int J Appl Sci Tech. 3(5): 107-115.

Abd-El-Ghany ME, Ammar MS and Hegazy AE (2010), Use of olive waste cake extract as a natural antioxidant for improving the stability of heated sunflower oil, World Appl Sci J. 11(1): 106-113.

Augustyniak A, Bartosz G, Cipak A, Duburs G, Horakova L, Luczaj W, Majekova M, Odysseos AD, Rackova L, Skrzydlewska E, Stefek M, Strosova M, Tirzitis G, Venskutonis PR, Viskupicova J, Vraka PS and Zarkovic N (2010), Natural and synthetic antioxidants: an updated overview, Free Radic Res. 44(10): 1216-1262. DOI: org/10.3109/10715762.2010.508495

Aluyor EO and Ori-Jesu M (2008), The use of antioxidants in vegetable oils - A review, Afr J Biotech. 7(25): 4836-4842.

Anwar F, Bhanger MI and Yasmeen S (2003), Antioxidant activity of some natural extracts in corn oil In: Advanced Research of Plant Lipids, Proceeding of 15-ISPL, Okazaki, Japan, pp 24-30.

Bandak G, Dermesonlouglou EK, Taoukis PS and Oreopoulou V (2011), Antioxidant effect of Majorana syriaca extract in bulk corn oil and o/w emulsion after applying high hydrostatic pressure, Food Chem. 125: 1166-1170. DOI: org/10.1016/j.foodchem.2010.10.022

Chatha SAS, Anwar F, Manzoor M and Bajwa JR (2006), Evaluation of the antioxidant activity of rice bran extracts using different antioxidant assays, Grassay Aceites. 57(3): 328-335.

Gertz C, Klostermann S and Kochhar SP (2000), Testing and comparing oxidative stability of vegetable oils and fats at frying temperature, Eur J Lipid Sci Tech. 102: 543-551.

Iqbal S and Bhanger MI (2007), Stabilization of sunflower oil by garlic extract during accelerated storage, Food Chem. 100(1): 246-254.

International Union of Pure and Applied Chemistry (IUPAC) (1987), Standard methods for the analysis of oils, fats and derivatives, $7^{\text {th }}$ Rev. Ed. Paquot $\mathrm{C}$ and Hautfenne $\mathrm{A}$, Blackwell Scientific, London

Ko SH, Park JH, Kim SY, Lee SW, Chun SS and Park E (2014), Antioxidant effects of spinach (Spinacia oleracea L.) supplementation in hyperlipidemic rats, Prev Nutr Food Sci. 19(1):19-26. DOI: 10.3746/pnf.2014.19.1.019 
Karoui IJ, Dhifi W, Jemia MB and Marzouk B (2011), Thermal stability of corn oil flavoured with Thymus capitatus under heating and deep-frying conditions, J Sci Food Agri. 91(5): 927-933.

Kanner J (2007), Dietary advanced lipid oxidation end products are risk factors to human health, Mol Nutr Food Res. 51: 1094-1101.

Kris-Etherton PM, Hecker K, Banamome A (2002), Bioactive compounds in foods: their role in the prevention of cardiovascular disease and cancer, Am J Med. 113: 71-88. DOI: org/10.1016/S0002-9343(01)00995-0

Li S, Chen G, Zhang C, Wu M, Wu S and Liu Q (2014), Research progress of natural antioxidants in foods for the treatment of diseases, Food Sci. Human Wellness 3(3-4): 110-116.

Lee J, Lee S, Lee H, Park K and Choe E (2002), Spinach (Spinacia oleracea) powder as a natural food-grade antioxidant in deep-fat-fried products, J Agri Food Chem. 50: 5664-5669.

Nosratpour M, Farhoosh R and Sharif A (2017), Quantitative indices of the oxidizability of fatty acid compositions, Eur $J$ Lipid Sci. Tech. 119: 1700203.

Navas PB, Duran AC and Flores I (2006), Effect of black tea, garlic and onion on corn oil stability and fatty acid composition under accelerated oxidation, Int J Food Sci. Tech. 41: 243-247. DOI: org/10.1111/j. 13652621.2005.01056.x

Naz S, Siddiqi R, Sheikh H and Sayeed AS (2005), Deterioration of olive, corn and soybean oils due to air, light, heat and deep-frying, Food Sci. Int. 38(2): 127-134.

Naz S, Sheikh H, Siddiqi R and Sayeed AS (2004), Oxidative stability of olive, corn and soybean oil under different conditions, Food Chem. 88: 253-259.

Redondo-Cuevas L, Castellano G and Raikos V (2017), Natural antioxidants from herbs and spices improve the oxidative stability and frying performance of vegetable oils, Int J Food Sci. Tech. 52(11): 2422-2428.

Rajendran P, Nandakumar N, Rengarajan T,Palaniswami R, Gnanadhas EN, Lakshminarasaiah U, Gopas J and Nishigaki I (2014), Antioxidants and human diseases, Clinica Chimica Acta. 436: 332-347.

Ramadan MF and Wahdan KMM (2012), Blending of corn oil with black cumin (Nigella sativa) and coriander
(Coriandrum sativum) seed oils: Impact on functionality, stability and radical scavenging activity, Food Chem. 132(2): $\quad 873-879$. DOI: org/10.1016/j.foodchem.2011.11.054

Shahidi F and Zhong Y (2010), Lipid oxidation and improving the oxidative stability, Chem Soc Rev. 39: 4067-4079.

Sultana B, Anwar F, Asi MR and Chatha SAS (2008), Antioxidant potential of extracts from different agro wastes: Stabilization of corn oil, J Food Chem. 59(3): 205-217.

Saha MR, Alam MA, Akter R and Jahangir R (2008), In vitro free radical scavenging activity of Ixora coccinea $\mathrm{L}$, Bangladesh $J$ Pharmacol. 3: 90-96. DOI: org/10.3329/bjp.v3i2.838

Sultana B, Anwar F and Przybylski R (2007), Antioxidant potential of corncob extracts for stabilization of corn oil subjected to microwave heating, Food Chem. 104: 997-1005.

Siddiq A, Anwar F, Manzoor M and Fatima M (2005), Antioxidant activity of different solvent extracts of Moringa Oleifera leaves under accelerated storage conditions of sunflower oil, Asian J Plant Sci. 4(6): 630-635.

Steel RGD, Torrie JH and Dicky DA(1997), Principles and procedures of statistics. a biometrical approach, $3^{\text {rd }}$ Ed., McGraw Hill Book Co. Inc., New York.

Tundis R, Tenuta MC, Loizzo MR, Bonesi M, Menichini F and Duthie G (2017), Natural compounds and vegetable powders improve the stability and antioxidant properties of Brassica napus L. var. oleifera (rapeseed) oil, Eur J Lipid Sci Tech. 119(4): 1-11. DOI: org/10.1002/ejlt.201600228

Taghvaei M and Jafari SM (2015), Application and stability of natural antioxidants in edible oils in order to substitute synthetic additives, J Food Sci Tech. 52(3): 1272-1282.

Tehseen M, Hina S, Nisa A and Ahmad A (2014), Antioxidant Potential of Differently Irrigated Soil Grown Varieties of Spinach, World Appl Sci J. 32 (7): 1235-1241.

Ullah J, Hamayoun M, Ahmad T, Ayub M and Zarafullah M (2003), Effect of light, natural and synthetic antioxidants on stability of edible oil and fats, Asian $J$ Plant Sci. 2(17-24): 1192-1194. 